\title{
EL ÚLTIMO PROYECTO DEL GATEPAC. EL PROYECTO DE SIXTO ILLESCAS PARA EL CONCURSO DE ENSANCHE DE A CORUÑA EN 1940
}

Francisco Dinís Díaz Gallego

Universidade da Coruña

Data recepción: 2017/01/29

Data aceptación: 2017/04/28

Contacto autor: franciscodiazgallego@outlook.es

ORCID: https://orcid.org/0000-0003-0362-6212

\section{RESUMEN}

En el presente artículo se analiza el proyecto realizado por el arquitecto catalán, miembro del GATEPAC, Sixto Illescas para el Concurso de Proyectos de Ensanche de A Coruña, convocado en febrero de 1940 por el alcalde José Pérez-Ardá a fin de satisfacer las necesidades de expansión de la ciudad y realizar dos proyectos clave en las primeras décadas del siglo XX: el Estadio Municipal de Riazor y el Parque Joaquín Costa en el monte Santa Margarita. Los equipos participantes serán fiel reflejo del estado del urbanismo de posguerra y del resultado del Concurso y la composición de su jurado saldrá el que será el primer Plan General de la ciudad, el Plan Cort de 1945. La obra del arquitecto catalán constituye la única propuesta conservada de dicho concurso, y se podría decir que se trata del último proyecto del GATEPAC.

Palabras clave: GATEPAC, urbanismo s.XX, movimiento moderno, zonificación, A Coruña

\section{ABSTRACT}

This article analyzes the project carried out by the Catalan architect and GATEPAC member, Sixto Illescas, for the Ensanche de A Coruña Design Contest, announced in February 1940 by the mayor, José Pérez-Ardá, to meet the city's need for expansion and undertake two key projects of the time: the Estadio Municipal de Riazor and the Parque Joaquín Costa, on Monte Santa Margarita. The teams that took part in the projects were a faithful reflection of the state of town planning in the post-Spanish civil war period, and both the contest and the composition of the jury would result in the first city's master plan: the Plan Cort of 1945. The Catalan architect's design is the only one still standing from this contest, and it can be described as the final GATEPAC project.

Keywords: GATEPAC, 20th century town planning, the modern movement, zoning, Corunna

\section{La situación urbanística en la ciudad de los años 30}

Los años 30 serán en la gran mayoría de las ciudades españolas una década plagada de proyectos de expansión y planificación urbanística, amparados por la aprobación del Estatuto Municipal la década anterior. Los principales arquitectos españoles, con el apoyo de los gobiernos municipales y central, buscan dar solución al crecimiento de las principales capitales de provincia. Así, en Galicia, será en esta época cuando se planteen dos documentos que no llegarían a aprobarse: el Plan para Vigo (1932) de Antonio Palacios Ramilo (1874-1945) y el Plan de Reforma y Ensanche de 


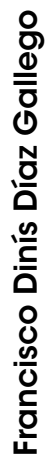

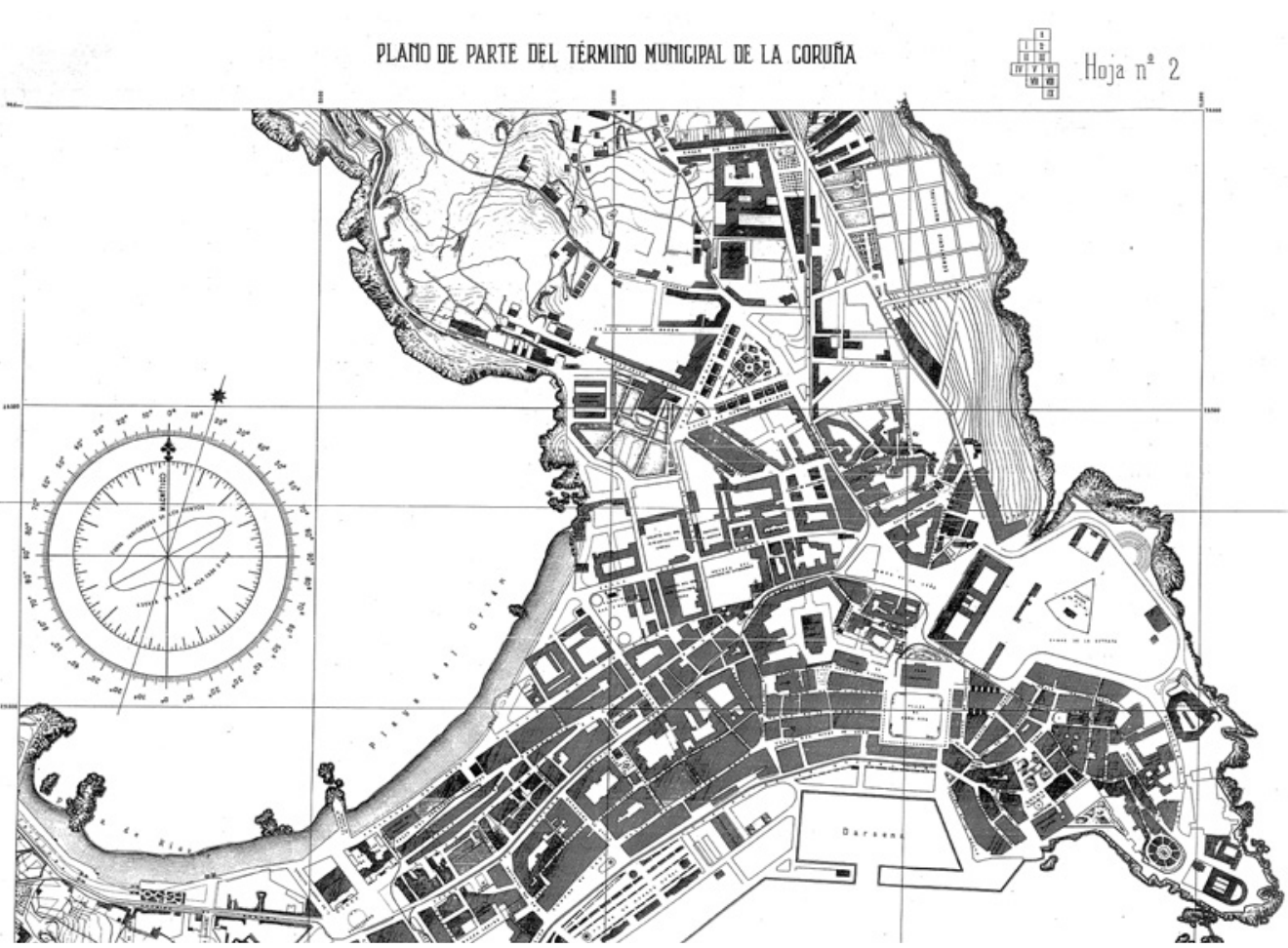

Fig. 1. Fragmento del Plano de A Coruña del ingeniero militar Fermín Gutiérrez (1931). Fuente: Archivo Municipal Urbanismo, A Coruña

Ferrol (1930) de Santiago Rey Pedreira (19021977); el primero, anulado en 1939, aportaba una importante visión metropolitana y de zonificación de la ciudad, pero pecaba de historicista al continuar con las ideas urbanísticas del siglo XIX. El segundo, que se paralizará con el inicio de la guerra civil, se sitúa en el contexto europeo de la época en consonancia con documentos como el Plan Berlage para Ámsterdam de 1917 o los proyectos urbanísticos de Jacobus Oud o Tony Garnier.

En A Coruña, la elaboración en el año 1931 de una nueva base cartográfica de la mano del ingeniero militar Fermín Gutiérrez de Soto (18971969) supuso para el futuro desarrollo de la ciudad un impacto similar al que tuvo en 1874 el plano de Barón y Yáñez sobre el cual se proyectaron los ensanches de Juan Ciórraga y Fernández de la Bastida (1836-1891) y de Pedro Mariño y Ortega (1865-1931), y los proyectos de alineaciones de calles en el barrio de la Pescadería. El plano de Fermín Gutiérrez nos muestra la traza de los dos ensanches parcialmente edificados, así como el desarrollo de la Ciudad Jardín y los arrabales de Cuatro Caminos, los Mallos y Santa Lucía. Pero el principal interés del plano no radica en las trazas de la ciudad consolidada sino en el detalle que aporta del resto del término municipal y del contacto entre las zonas urbanas y las rurales. Parcelario agrícola, núcleos rurales y caminos aparecen reflejados con gran definición, lo cual será clave para los planteamientos de crecimiento de la ciudad en los años posteriores. La realización del plano de Fermín Gutiérrez visualiza, en definitiva, las áreas de oportunidad de la nueva ciudad y la necesidad de ordenación de todo el término municipal (fig. 1).

En el año 1935 aparecerán las primeras noticias de intentos del gobierno central por apoyar económicamente un documento urbanístico que ordene más allá del ya sobrepasado Ensanche de Mariño y Pan de Soraluce, sin que se conserve ningún documento al respecto de tales iniciativas 
urbanísticas. Dichos intentos se verán truncados por la guerra civil (fig. 2).

No será hasta 1937 cuando, apoyándose en el Estatuto Municipal', se establezcan las primeras orientaciones urbanísticas sobre el futuro de la ciudad, tanto la construida, como la que tendrá que construirse en los años sucesivos. Así, se divide A Coruña en cuatro sectores para los cuales habrán de establecerse ordenanzas de construcción diferentes: Interior, Ensanche, Zona de Contacto y Extrarradio. De las cuatro zonas las dos primeras contaban ya con ordenanzas de construcción propias, siendo de mayor urgencia establecerlas para las dos siguientes. Todos los esfuerzos de expansión de la ciudad se centrarán en la zona de la Gaiteira, un populoso núcleo tradicional situado en la rada interior del puerto, pero más allá de la zona industrial de la Palloza y de las vías del tren.

\section{Pérez-Ardá, el alcalde-urbanista}

El abogado José Pérez-Ardá (1913-1978) asume a los 26 años el bastón de mando como primer alcalde de la ciudad después de la guerra civil. Su primer cargo público le había llegado en 1938, siendo ponente de urbanismo durante el gobierno de Fernando Álvarez de Sotomayor (1875-1960). Desde dicho cargo retomará una serie de viejos proyectos urbanos tanto de expansión como de actuaciones sobre lo ya construido, iniciándose con el Paseo de Riazor, la urbanización de las calles Linares Rivas y Primo de Rivera, los planos de alineaciones para Castiñeiras, Santa Lucía y Fernández Latorre, y la apertura de la calle Durán Loriga.

Pero será a la llegada a la alcaldía, en 1939, cuando asuma sus proyectos más ambiciosos. Pese a la brevedad de sus mandatos², su implicación en la política urbanística de la ciudad fue relevante, por lo cual resulta sorprendente que haya quedado al margen de todos los análisis realizados hasta la fecha.

El interés por la realización de una política urbanística proactiva desde la administración municipal le llevó, al inicio de su mandato, en 1939, a plantear la expropiación de la totalidad del término municipal para evitar la especulación urbanística. Dicha actuación venía siendo defen-

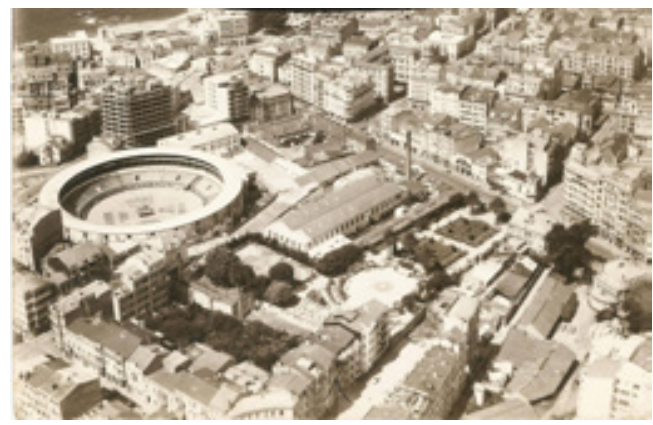

Fig. 2. Imagen del Ensanche de A Coruña - Web "Coruña Fotos Antiguas"

dida por urbanistas del movimiento moderno en los años anteriores; en 1932, el arquitecto Fernando García Mercadal (1896-1985), miembro del GATEPAC, en una conferencia en Santander defendía que mientras no fuese "abolida la propiedad del suelo en la ciudad, nacionalizada o municipalizada, el urbanismo encontrará siempre un enorme obstáculo"s; y en el Congreso Municipalista de Gijón del año 1934 dos de las tres ponencias relativas a urbanismo se titulaban: "Urbanismo y expropiación forzosa" y "Bases de expropiación forzosa". Ya en plena posguerra, en la primera Asamblea Nacional de Arquitectos, el arquitecto y urbanista falangista Pedro Bidagor Lasarte (1906-1996) alabará la expropiación como método de freno de la especulación en su ponencia "Plan de Ciudades", donde se expuso el modelo de Ciudad del Movimiento, del "Orden Nuevo" ${ }^{4}$.

Aunque la propuesta del alcalde Pérez-Ardá no llegó a ser ejecutada, sí enunciaba una interesante visión que intentaba anticiparse a los males urbanísticos posteriores de los años 50 y 60, los cuales afectarían duramente a la ciudad. A la fallida idea de expropiación del término municipal le seguirán durante su primer año de mandato proyectos urbanísticos ambiciosos como la creación de un gran parque exterior situado sobre los montes de Elviña y Zapateira que "cerrase" la ciudad y sus focos perspectivos, siguiendo la idea de ciudad falangista, cerrada y finita, que tiene su modelo en la Salamanca (1939) de Victor D’Ors (1909-1994) y adelantando la propuesta del gran parque a cota $+100 \mathrm{~m}$ ideada por el urbanista Joan Busquets en su revisión del Plan General de Ordenación Municipal para A Coruña del año 
2013. También resultaban relevantes el proyecto de "City Park" (sic) en la colina de Santa Margarita para el que proyectaba un funicular y una gran avenida que lo conectase con la Estación de Tren de Santiago, una gran plaza en el entorno de la actual Plaza de Cuatro Caminos; grandes equipamientos públicos y el que sería su empeño personal, el nuevo Stadium de Riazor.

\section{El Concurso de Ensanche de $\mathbf{1 9 4 0}$}

Consciente de la necesidad de un nuevo instrumento urbanístico que le permitiese llevar a cabo los proyectos anunciados, el 20 de febrero de 1940 el Pleno aprueba una proposición de alcaldía para la convocatoria de un Concurso de Proyectos de formación del nuevo plano de Ensanche, de extrarradio y de reforma interior de la ciudad, siendo el texto con el que Pérez-Ardá da inicio al proceso otra muestra más de la importancia que iba a tener en sus decisiones su visión acerca del urbanismo5.

Las bases del concurso, redactadas por el arquitecto municipal Santiago Rey Pedreira6, figura clave del racionalismo en Galicia; no fijan un ámbito de crecimiento de la ciudad ni una extensión máxima, dejando dichas decisiones en manos de las propuestas de los técnicos; si bien estipulan que se deben evitar zonas del extrarradio ya edificadas. Por otro lado, sí se establece la necesidad de que la propuesta concilie los tres aspectos fundamentales de la ciudad actual, que según la corporación son sus vocaciones marítima, industrial y veraniega. Se fija un ancho mínimo de las calles de 20 metros, exigiendo garantizar la conexión del nuevo desarrollo con la trama urbana existente; y se enumeran una serie de nuevos equipamientos.

También aparece en las bases un viejo proyecto que irá reapareciendo en los años sucesivos, la idea de una zona residencial y de ocio vinculada a las playas de Santa Cristina y a la Ría del Burgo, una "Ciudad Jardín" que habría de estar bien comunicada con la ciudad y que abarcaría desde el Puente Pasaje a San Pedro de Nós, en terrenos del ayuntamiento de Oleiros, y por tanto en una visión alargada del territorio municipal similar a la visión metropolitana de Palacios en su Plan para Vigo. Esta intervención volverá en los años 60 de la mano de Andrés Fernández-Albalat Lois y
Milagros Rey Hombre con su proyecto de zona residencial en Santa Cristina enfocada al turismo internacional.

La convocatoria del Concurso de Proyectos de formación del nuevo plano de Ensanche, de extrarradio y de reforma interior de la ciudad vuelve a situar, desde los proyectos de Ensanche de finales del XIX, al urbanismo en el centro del debate político de la ciudad, y reúne en el mismo jurado al Director General de Arquitectura, Pedro Muguruza Otaño (1893-1952), al urbanista César Cort Botí (1893-1978) y al arquitecto municipal Santiago Rey Pedreira (1902-1977). Las peticiones de documentación de los estudios interesados en participar en el concurso, conservadas en el Archivo Histórico Municipal de A Coruña, también demuestran el interés suscitado en el proyecto de Ensanche, estando presentes todos los arquitectos de relevancia de la España de posguerra: Gabriel Alomar Esteve, Fernando Moreno Barberá, Pedro Bidagor Lasarte, José Fonseca Llamedo, Luis Moya Blanco o Gaspar Blein Zarazaga entre otros.

Diversos problemas relacionados con falta de documentación y las negativas del ayuntamiento a ampliar el plazo de presentación de propuestas, menguaron finalmente los proyectos presentados; limitándose a cuatro de los veintiocho que inicialmente lo habían solicitado. Este hecho, que hizo caerse del concurso a los principales urbanistas del nuevo régimen fue criticado con dureza en el propio fallo del jurado?. Los equipos participantes serían: Sixto Illescas i Mirosa con Joaquín Fernández Marqués; José María Barenys Gambús con Ernesto Paradell García; Joaquín García Alcañíz con Pere Pigrau Casals y un único equipo coruñés formado por Ramiro Mariño Caruncho, Juan Martínez Barbeito y Pablo Iglesias Atocha. Resulta llamativa la composición de los equipos participantes, estando tres de ellos conformados por arquitectos catalanes de diversa procedencia y un único equipo local.

La ausencia hasta hoy de la documentación aportada por los técnicos concursantes nos hacía imposible imaginar la ciudad planteada en las diferentes propuestas presentadas. El hallazgo en el Archivo del Collegi de Arquitectes de Catalunya del proyecto del miembro del GATEPAC ${ }^{8}$ Sixto 
Illescas (1903-1986) nos permite conocer en detalle al menos una de ellas.

\section{El proyecto de Illescas para A Coruña}

El proyecto entregado por el tándem Illescas/ Fernández-Marqués es el primero que el arquitecto catalán firma tras la finalización de la guerra civil y fue realizado en pleno proceso de su inhabilitación, que culminaría un año después. Dicho hecho solo tiene explicación entendiendo las singularidades de la liberal sociedad coruñesa en la que al mismo tiempo dos arquitectos de gran significación republicana, Santiago Rey Pedreira y Antonio Tenreiro Rodríguez, continuaban con su labor profesional, protegidos por el alcalde Pérez Ardá en el caso del primero, que continuaba como arquitecto municipal; y por las familias Pastor y Barrié de la Maza el segundo, inhabilitado para cargos públicos. Dos años antes, en 1938, otro hecho insólito confirma esta excepción, el arquitecto catalán Jordi Tell Novellas, apresado por la Gestapo y devuelto a España, proyectaba, recluido en Coruña, una vivienda de veraneo para la familia de uno de los principales industriales de la ciudad, Emilio Cervigón.

Joaquín Fernández Marqués por su parte, era en aquellos años un joven arquitecto formado en el periodo republicano que había participado siete años antes, junto con otros arquitectos y estudiantes de arquitectura, en el conocido como "Crucero universitario por el Mediterráneo de $1933^{\prime \prime}$ " un viaje de estudios impulsado por Manuel García Morente y Fernando de los Ríos, en el cual participaron arquitectos como Pascual Bravo Sanfeliú y estudiantes de arquitectura como Fernando Chueca Goitia.

Se trataba por tanto de un equipo formado por uno de los principales referentes de la arquitectura republicana, miembro del GATCPAC, y un arquitecto formado durante la República, participante activo en sus nuevos modelos pedagógicos.

Este proyecto se trataría del segundo proyecto urbanístico abordado por Sixto Illescas, que en 1934 había proyectado el sector "Barcelona Playa" en Prat de Llobregat siguiendo las directrices de la Ciutat del Repós i de Vacances del GATCPAC (1932-1935).

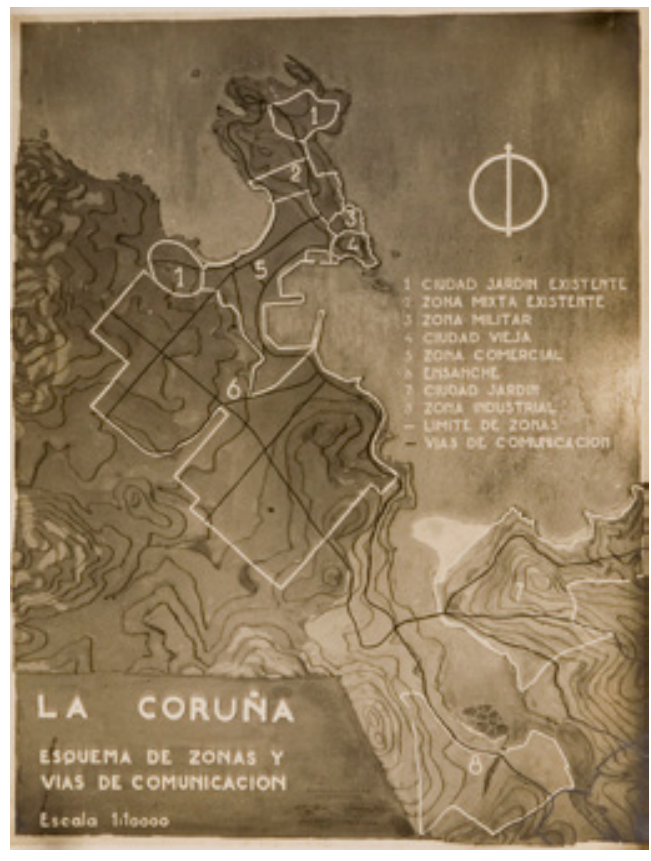

Fig. 3. Esquema de zonas Concurso de Ensanche de A Coruña 1940. Fuente: Fondo Illescas, Archivo del Collegi de Arquitectes de Catalunya

En carta remitida al ayuntamiento de A Coruña por el propio Sixto Illescas con fecha de 28 de agosto de $1940^{10}$ se hace referencia a la documentación aportada para participar en el concurso: veintidós planos, dos maquetas y un documento de memoria, de la que solo se conserva parte. El expediente archivado se divide en dos, un primer lote relacionado con los trabajos previos al proyecto de ensanche (bases del Concurso, información sobre precios de expropiación, datos turísticos de la ciudad, un Anuario de la Editorial Nós, varias guías turísticas en diferentes idiomas y 20 postales de época) y un segundo lote compuesto por un plano de zonificación, una ordenación a mano alzada, fotos de una maqueta, varias vistas perspectivas, y planos de detalle de las manzanas tipo y la llamada "Plaza Popular", punto central del nuevo desarrollo. También se conservan secciones del terreno con el viario propuesto y estudios de soleamiento (fig. 3).

Lo primero a destacar es el hecho de que gran parte de la base cartográfica no corresponde con los planos de Fermín Gutiérrez de 1931 sino que se trata de juegos de planos temáticos (co- 


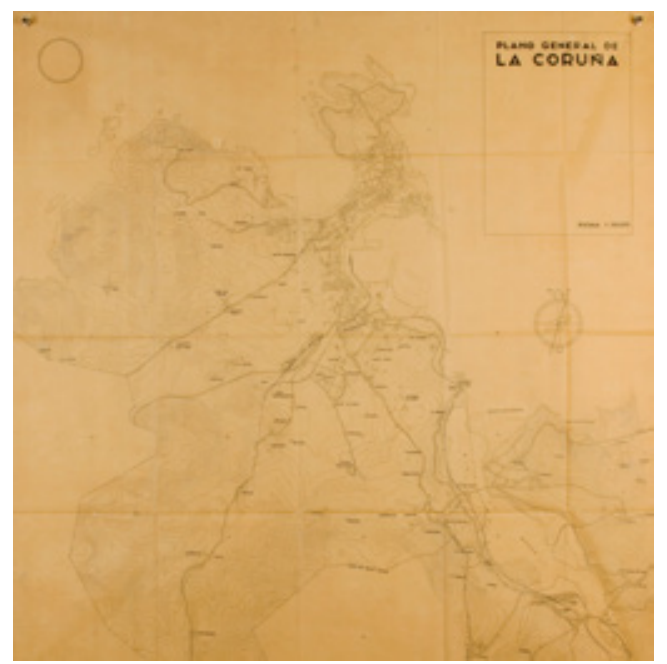

Fig. 4. Base Cartográfica Concurso de Ensanche, fechada en 1935. Fuente: Fondo Illescas, Archivo del Collegi de Arquitectes de Catalunya

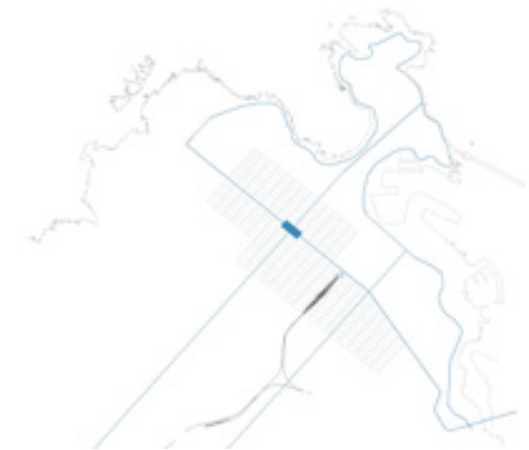

Fig. 5. Esquema viario del proyecto de Ensanche de Sixto Illescas para A Coruña. Fuente: elaboración propia

municaciones, antigüedad de la edificación,...) fechados en 1935, lo cual podría confirmar los intentos del gobierno republicano por dotar a la ciudad de un nuevo documento urbanístico, que en cualquier caso se vio truncado por la guerra civil (fig. 4).

La ciudad imaginada por Illescas sobrepasa sus límites municipales, extendiéndose por la costa de Oleiros hasta Bastiagueiro siguiendo modelos de ciudad jardín, y por los ayuntamientos limítrofes de Culleredo y Cambre hasta la cola de la ría, donde ubica la zona industrial y los equipamientos que debían alejarse del centro tales como el matadero municipal, en aquella época en el Orzán. Será el primer documento urbanístico en el que se entienda la ordenación de la ciudad con una visión metropolitana, exigencia definida en las propias bases del concurso, similar a la del Plan Palacios de Vigo de los años 30. Ninguno de los documentos urbanísticos posteriores abordará la ciudad más allá de sus límites administrativos hasta el Plan Comarcal Ciudad de las Rías de Andrés Fernández-Albalat Lois a finales de los años 60.

\section{El esquema viario del nuevo Ensanche}

El planteamiento viario de Sixto Illescas para A Coruña es netamente moderno, organizándose a partir de cinco modelos de vías según la sección de los mismos y relevancia funcional dentro de la red viaria del nuevo desarrollo. Las vías de mayor sección, denominadas "Gran Vía" en los planos del proyecto, varían entre los 60 metros de las vías tipo $A$ y $B$, y los 40 metros de las vías tipo $C$. El siguiente escalón lo conforman las vías que delimitan los diferentes "barrios" o agrupamientos residenciales, llamadas vías tipo $\mathrm{D}$, con una sección de 30 metros de ancho. Los viales entre bloques, el tipo $E$, toman como sección el ancho mínimo marcado por las bases del concurso, 20 metros. Finalmente, la red viaria se completa con viales semi-peatonales interiores a los bloques residenciales con una sección de 5 metros. En cualquiera de los viales definidos, es una invariante el porcentaje de sección dedicado al peatón/arbolado frente al dedicado al tráfico rodado, manteniendo siempre un equilibrio de 50-50. Este equilibrio está muy alejado del de la ciudad actual, situado en un 30-70 a favor del espacio dedicado al tráfico rodado; pero sí se da en proyectos como el Eixample de Barcelona, donde la sección tipo de 20 metros dedica 10 metros a espacio peatonal y 10 metros a la circulación de vehículos (fig. 5).

Las tres "Gran Vías" definidas en el proyecto de Ensanche conforman un eje viario en " $\mathrm{H}$ " que tiene como punto central la Estación de Santiago (actual Estación de San Cristóbal) y como elemento representativo la llamada Plaza Popular situada en el punto de mayor altitud y en el cruce de dos de ellas, un nuevo foco de centralidad donde Illescas sitúa los nuevos edificios del gobierno municipal, hoteles, edificios comerciales y el Palacio de Justicia. 
El desarrollo viario perfectamente rectilíneo y rotundo cose en sus extremos exteriores los 3 accesos principales a la ciudad: la carretera de Fisterra, la de Santiago por Mesoiro y el Puente del Pasaje con el centro de la ciudad a través de una vía de circunvalación de la península propuesta en el proyecto, y que aparecerá también en los documentos urbanísticos posteriores (Cort 1945, Iglesias Atocha 1948) y a través de un vial que comunica la Plaza de Pontevedra con Zalaeta fruto del ensanchamiento de la calle San Andrés.

\section{Zonificación y definición del esquema de crecimiento}

El ensanche propuesto por Illescas entiende el nuevo desarrollo como una nueva ciudad y no como una ampliación de la existente, alejándose del modesto planteamiento del Ensanche de Mariño y Pan de Soraluce, y acercándose a las ideas del Ensanche de Cerdá de Barcelona y su versión moderna, el Plan Macià (fig. 6).

Con el Plan Macià, elaborado por arquitectos GATCPAC, grupo del que formaba parte Illescas, comparte además las principales ideas de actuación: saneamiento de la ciudad existente, algo no planteado en las bases del concurso pero asumido como necesario por el arquitecto catalán; fin del crecimiento de la ciudad actual, el nuevo desarrollo encorseta la ciudad central e impide que se siga desarrollando al margen de lo estipulado por el nuevo plan de ensanche; zonificación rigurosa, y unión de la ciudad y el mar, algo presente en el proyecto de Sixto Illescas tanto a través de las zonas verdes de borde marítimo como del vacío proyectado frente a la Estación de tren de Santiago.

Illescas se apoya en la retícula viaria en " $\mathrm{H}$ " y sus ejes noroeste-sureste y noreste-suroeste, y define unas manzanas de 160x60 m rompedoras formal y funcionalmente al optar por bloques estrechos y exentos con viviendas pasantes completamente exteriores, descartando las manzanas cerradas y el uso de patios interiores. Los bloques de 10 metros de crujía y 6 pisos de altura se apoyan sobre un zócalo comercial de 30 metros de crujía, formalizando volúmenes en forma de " $L$ " tanto en planta como en sección (fig. 7).

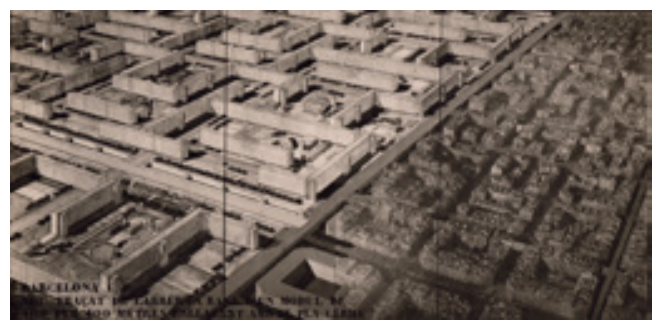

Fig. 6. Fotomontaje original del Plan Maciá, 1934. Fuente: web Urban Networks

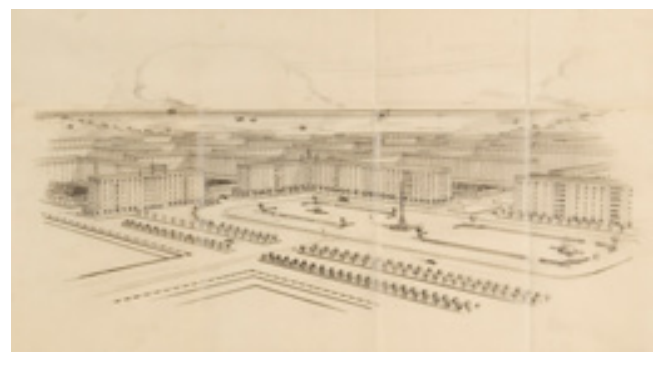

Fig. 7. Vista del proyecto de Ensanche para A Coruña de Sixto Illescas, 1940. Fuente: Fondo Illescas, Archivo del Collegi de Arquitectes de Catalunya

El espacio restante de la manzana será siempre espacio público, variando su uso según la manzana: desde equipamientos locales tales como escuelas infantiles, salas de lectura, grupos escolares o servicios municipales, hasta zonas verdes y de esparcimiento. Esta conformación de la manzana produce una radical inversión de las proporciones de espacio público y privado con respecto a la ciudad preexistente. Frente a la manzana de Ensanche decimonónica, cuya ocupación es totalmente privada y el espacio público se obtiene de la extracción de manzanas de viviendas para la conformación de equipamientos y plazas; Illescas plantea una manzana en la que el $50 \%$ de la superficie es privada (zócalo comercial-viviendas) y un $50 \%$ es pública (equipamientos, viario de acceso y zonas verdes). Una vez más aparecen las referencias al Plan Macià y al único edificio construido acorde con las directrices del mismo, la Casa Bloc (1932-1936), en la que los bloques de viviendas rectilíneos se sitúan en torno a patios de manzana semiabiertos donde se ubican los servicios comunitarios.

Al margen de las zonas residenciales y de los equipamientos locales, el proyecto define los grandes ámbitos de equipamiento a nivel ge- 


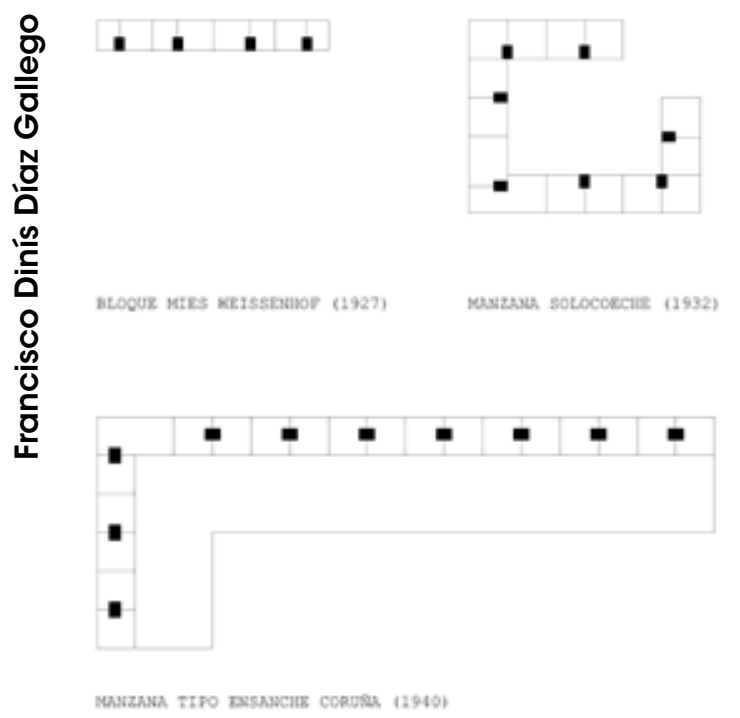

Fig. 8. Comparativa manzanas tipo: Ensanche de Illescas para A Coruña, Proyecto del GATEPAC para Concurso de Solocoeche en Bilbao (1932) y Bloque para la Weissenhof de Stuttgart, Mies van der Rohe (1927). Fuente: elaboración propia

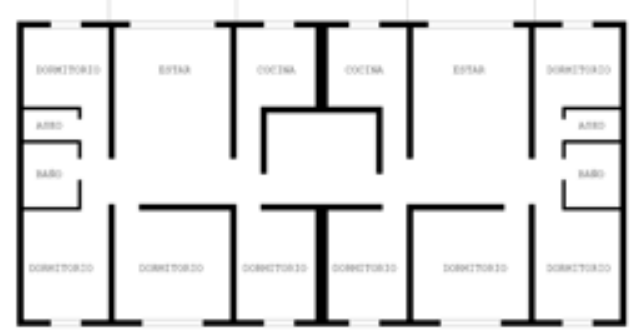

Fig. 9. La vivienda mínima del proyecto de Ensanche de Sixto Illescas para A Coruña (1940). Fuente: elaboración propia

neral, las zonas de trabajo e industria, y las de ciudad jardín, diferenciando por tanto las zonas según sus funciones. Tienen especial relevancia también los cuatro grandes parques definidos en el documento situados en A Silva, A Grela, Cuatro Caminos y todo el borde costero de la ría, a ambos lados de la misma.

\section{La vivienda tipo del nuevo Ensanche}

La definición de la manzana tipo del ensanche abarca también el proyecto de un modelo de vivienda para el nuevo desarrollo. La vivienda cuenta con todas las características de la vivienda moderna: doble crujía, totalmente exterior, estudios de soleamiento y un esquema de distribución que sitúa las zonas de día y de noche en diferentes orientaciones (fig. 9).

El análisis comparativo de la vivienda de Illescas con otros ejemplos proyectados por arquitectos del movimiento moderno europeo y el GATEPAC nos muestra los referentes que el arquitecto catalán tenía encima de su mesa de trabajo, siendo el proyecto de Mies van der Rohe para la Weisenhofsiedlung de Stuttgart el que presenta mayores similitudes. Curiosamente, 12 años antes, Illescas visitaría dentro de su viaje de fin de curso de la ETSAB el barrio de la ciudad alemana, volviendo del viaje con numerosos libros del proyecto y de obras de Hilberseimer"1 (fig. 8).

La vivienda tipo de Illescas es un cuadrado de diez por diez metros con salón, cocina independiente, baño y cuatro habitaciones. Además, cuentan todas con mirador. Aunque alejada de modelos más radicalmente modernos como las viviendas en dúplex del Plan Maciá o de proyectos europeos anteriores, propone en el contexto gallego de la época, un modelo nunca antes planteado con la excepción del Barrio de Recimil de Ferrol del arquitecto José Fonseca Llamedo, cuyo proyecto se inicia en 1939 y es inaugurado en 1944. Para encontrar soluciones residenciales similares habrá que esperar a los años 50 , con proyectos como el Barrio del Cristo de la Victoria de Vigo (1950) obra de Enrique Álvarez-Sala o el Grupo de Viviendas María Pita en A Coruña (1955) de Juan González Cebrián, que en cualquier caso no tienen la entidad del proyecto de Illescas ni en el tamaño de la actuación ni en cuanto al diseño de los espacios del barrio.

\section{La estética urbana del proyecto}

Pese a la modernidad de los planteamientos formales y funcionales de Illescas en su propuesta para el concurso, en los que están presentes todas las ideas del movimiento moderno español anterior a la guerra civil, el ambiente de posguerra y las directrices marcadas, primero por la reunión de arquitectos organizada por Pedro Muguruza en Burgos en febrero de 1939, y después por la Asamblea Nacional de Arquitectos organizada en Madrid por Pedro Bidagor en junio de ese mismo año; en las que se sentaron las bases de la "nueva 


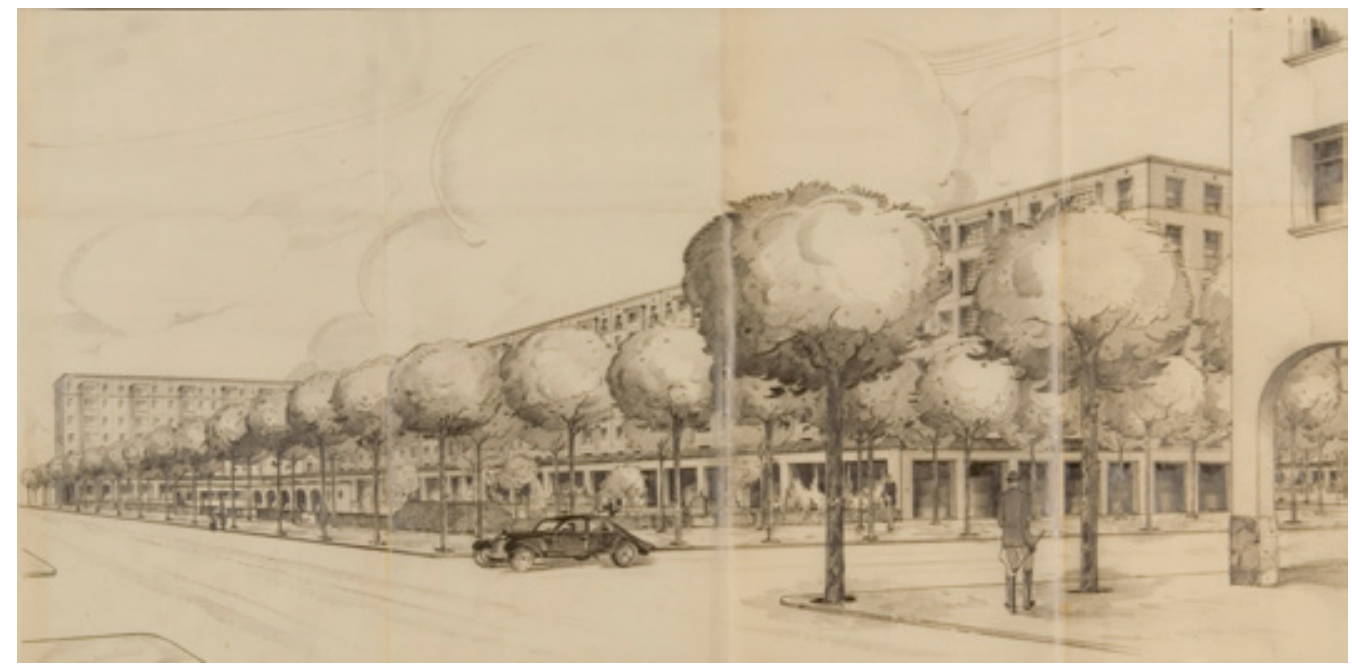

Fig. 10. Vista de una calle del proyecto de Ensanche de Sixto Illescas para A Coruña (1940). Fuente: Fondo Illescas, Archivo del Collegi de Arquitectes de Catalunya

Ciudad del Movimiento" 12 hacían inevitable optar por una estética del conjunto no sospechosa de pertenecer a la modernidad, y por tanto a los planteamientos del periodo de la Segunda República.

Resulta imposible entender el resultado del camuflaje estético proyectado por Illescas sin conocer su origen como estudiante en la Escuela de Arquitectura y sus referentes arquitectónicos más allá de los icónicos Le Corbusier y Hilberseimer. La Escuela de Arquitectura de Barcelona en la que se forma Illescas en los años 20 del pasado siglo se encontraba fuertemente dividida en dos grupos que pivotaban en torno a los principales arquitectos de la época. Por un lado, se encontraban los modernistas, inspirados por Puig i Cadafalch y Doménech i Muntaner, que representaban el ala conservadora de la escuela; y por otro los vinculados a la corriente del Noucentisme, entre los que se encontraban Bona, Florença y Azua, cuya obra se basaba en el estudio de las arquitecturas clásicas. Illescas, pese a renegar del encasillamiento estilístico de la escuela de la época decide, junto con sus futuros socios Sert y Torres Clavé, optar por las enseñanzas de los segundos, estudiando los órdenes clásicos y las obras del renacimiento catalán; culminando este estudio de la cultura clásica con un viaje por Italia donde visitan obras de Palladio, cuyo descubrimiento fue, en palabras del hijo de Illescas "su fulminante conversión a la arquitectura. A una arquitectura pura, contenida, sin gestos banales, no decorativa, regida por un orden comprensible y de una belleza fundamentada en las proporciones, la luz y la sombra"13

Quizás sería este viaje a Italia y el interés en la arquitectura de este país el que llevaría a Illescas a seguir con gran interés el racionalismo italiano y la obra del Gruppo 7, en especial la de los arquitectos Luigi Figini (1903-1984) y Gino Pollini (1903-1991).

Así, cuando llega la hora de formalizar estéticamente los bloques modernos de las manzanas del ensanche, Illescas opta por un lenguaje similar al del Racionalismo Italiano, donde la presencia de elementos clásicos como las arcadas desprovistas de toda decoración representa al mismo tiempo una vía más de la modernidad y un lenguaje aceptado por el "nuevo orden arquitectónico" de la dictadura franquista (fig. 10).

La apariencia de los bloques del nuevo ensanche es a pesar de todo fundamentalmente racionalista con fachadas carentes de decoración a excepción del último piso con un tratamiento de fachada diferenciado, miradores acristalados y soportales que alternan arcadas con dinteles rectos. Será en los edificios institucionales de la llamada Plaza Popular donde aparezcan mayores elementos decorativos, introduciendo estatuaria y huecos rematados en arco en el último piso. 
ㅇ En el diseño de los bloques está claramente presente la obra de Secundino Zuazo, con el que colaborarían arquitectos del GATEPAC como Fernando García Mercadal; que llega al racionalismo a través del estudio de las arquitecturas clásicas españolas.

\section{Conclusiones}

El proyecto de Ensanche para Coruña del GATEPAC Sixto Illescas constituye el único proyecto urbanístico de la modernidad en la ciudad, siendo sin duda un punto y aparte de la carrera del arquitecto catalán, que, a raíz de su inhabilitación, caminará hacia una conversión forzada a las nuevas tesis arquitectónicas de la posguerra.
Además, es el único documento que se conserva del Concurso convocado en 1940. Se trata de un proyecto que contiene tesis radicalmente modernas, inconcebibles en la España de la posguerra, y referencias claras a proyectos clave del movimiento moderno europeo y español camufladas con un lenguaje formal racionalista. Los modelos de crecimiento y espacios públicos proyectados hubiesen generado una ciudad ordenada, con gran presencia de espacios peatonales y verdes, y una red de equipamientos que no se conseguiría hasta muchas décadas después. El fallo del jurado del concurso ${ }^{14}$, que declara desierto el mismo, pone fin a esta ciudad moderna y abre paso al que será el otro gran documento urbanístico fallido de la Coruña del siglo XX, el Plan Cort de 1945'5. 


\section{NOTAS}

1 Fallo del Jurado del Concurso con fecha del 7 de octubre de 1940: [...] El Jurado juzga ser su obligación primera hacer resaltar la conducta ejemplar del Excmo. Ayuntamiento de La Coruña al convocar el concurso de proyectos de Ensanche de la ciudad, cumpliendo asi una disposición preceptiva del Estatuto Municipal, por un sistema que facilita al máximo grado la concurrencia de ideas generales y la conjunción de parciales aciertos en términos de liberalidad técnica, que debieran ser incentivo y motivo de atracción de los profesionales, al tiempo mismo, que de gratitud al haberse ofrecido ocasión inmejorable de prueba para sus aptitudes, logro de sus aspiraciones y motivo de contribución al mejoramiento de España. [...]

2 José Pérez-Ardá y López Valdivielso sería alcalde de A Coruña en los mandatos 1939-1941,1943-1944 y 1969-1974.

3 ARGÍZ VÁZQUEZ, Emilio El Ferrol que pudo haber sido, el Proyecto de Reforma y ensanche de la población de Ferrol, de Santiago Rey Pedreira (1930) (Anuario Brigantino 27, año 2004), 467-78.

${ }^{4}$ DE TERÁN, Fernando Planeamiento Urbano en la España Contemporánea. Historia de un Proceso Imposible (Editorial Gustavo Gili, Barcelona año 1978), 117

${ }^{5}$ Acta de la sesión del pleno del 20 de febrero de 1940 del Ayuntamiento de A Coruña:

[...] De muchos años atrás vinieron los Ayuntamientos gobernándose sin resolver este problema interesantísimo, más aún básico para conseguir el desarrollo normal de La Coruña. ¿Por qué causas no lo hicieron? No importa investigarlo, lo que urge es hacerlo. Por ello, interpretando así la intención de los señores Ediles, procuraré apartándome de problemas minúsculos o de interés particular, aunar los esfuerzos de los técnicos y gestores competentes para llegar a ultimar estas Bases [...] (Archivo Histórico Municipal de A Coruña)

${ }^{6}$ La autoría de las Bases del Concurso de Ensanche y Extensión de 1940 es atribuida al arquitecto municipal Santiago Rey Pedreira en la Tesis doctoral "SANTIAGO REY PEDREIRA. CONSTRUCTOR DE IDEAS", Luis Walter Muñoz Fontenla (2012)

7 Fallo del Jurado del Concurso con fecha del 7 de octubre de 1940: Paralelamente, $y$ en sentido inverso, debe lamentarse el Jurado de no encontrar una digna respuesta de la técnica, a tan estimable invitación, y censurar que se abstengan cómodamente del esfuerzo que supone una competición, quienes entre sus aspiraciones tienen la de seguir una línea ascendente en su crédito profesional y por su pretendida especialidad debieran contribuir con sus conocimientos al fin principal perseguido en el concurso que hoy se juzga, cual es el de lograr una acertada solución a uno de los problemas urbanísticos que más pueden atraer e interesar por sus características e importancia a los técnicos españoles.

8 El GATEPAC, Grupo de Artistas y Técnicos Españoles para el Progreso de la Arquitectura Contemporánea se dividía en 3 subgrupos: central, norte y oriental, siendo este último, llamado GATCPAC (Grup d'Artistes i Tècnics Catalans per al Progrès de l'Arquitectura Contemporània) al que pertenecía Sixto Illescas.

9 GRACIA ALONSO, Fernando El sueño de una generación: el crucero universitario por el Mediterráneo de 1933 Barcelona : Publicacions Edicions de la Universitat de Barcelona, [2006]

${ }^{10}$ Documentación Concurso de Ensanche 1940 conservada en el Archivo Histórico Municipal de A Coruña. (Signatura 8079.03)

${ }^{11}$ ILLESCAS DE LA MORENA, Albert Sixte Illescas, Arquitecte. De la vanguardia al olvido (Collegi de Arquitectes de Catalunya, año 2004),93.

${ }^{12}$ DE TERAN, Fernando. Planeamiento Urbano en la España Contemporánea Historia de un Proceso Imposible, (1 ${ }^{\text {a }}$ Ed. Gustavo Gili, Barcelona. 1978), 124.

${ }^{13}$ ILLESCAS DE LA MORENA, Albert Sixte Illescas, Arquitecte. De la vanguardia al olvido (Collegi de Arquitectes de Catalunya, año 2004),85.

${ }^{14}$ Fallo completo del Jurado del Concurso con fecha del 7 de octubre de 1940: En la ciudad de La Coruña a siete de octubre de mil novecientos cuarenta, siendo las seis horas de la tarde, en las Casas Consistoriales de esta ciudad, se reunieron el Excmo. Señor Director General de Arquitectura D. Pedro Muguruza; Ilmo. Señor Fiscal Provincial de la Vivienda D. Juan Teijeiro Flores en representación del Excmo. Señor Fiscal Superior de la Vivienda; el Sr. Alcalde interino del Excmo. Ayuntamiento de La Coruña, D. Ramón de Soto Lemos; Sr. Teniente Alcalde Ponente en Obras Municipales D. Andrés Reboredo; Sr. D. César Cort en representación de la Escuela Superior de Arquitectura de Madrid, Sr. D. Javier Sanz en representación del Colegio de Arquitectura de León; Sr. D. Víctor Solórzano. Ingeniero Municipal y el Sr. D. Santiago Rey Pedreira Arquitecto Municipal, y con las ausencias del Excmo. Sr. D. José Palanca de cuya representación y voto está delegado $D$. César Cort; y los Sres. D. Antonio Palacios representante de la Academia de Bellas Artes y D. Amadeo Llopart representante de la Escuela Superior de Arquitectura de Barcelona quienes delegan, como en el caso precedente en el Excmo. Señor Director General de Arquitectura D. Pedro Muguruza, todos los cuales en acuerdo unánime resuelven emitir el siguiente fallo en funciones de Jurado Calificador del Concurso de Proyectos para la ampliación de la zona de Ensanche de La Coruña, sobre los cuatro proyectos presentados por $D$. Sixto Illescas y D. Joaquín Fernández Marqués; D. Ernesto Paradell García y D. J.M. Barenys; D. Ramiro Mariño Caruncho, D. Juan Martínez Barbeito y D. Pablo Iglesias Atocha; y D. Joaquín García Alcañiz y D. Pedro Pigrau. De este último proyecto, por demorarse su llegada, no tuvo conocimiento el Jurado en su primera reunión, pero habiendo acompañado justificación suficiente con talón de F.C. con fecha de imposición de siete de septiembre de mil novecientos cuarenta, en Barcelona, se considera con arreglo a las bases del concurso como admitido

El Jurado juzga ser su obligación primera hacer resaltar la conducta ejemplar del Excmo. Ayuntamiento de La Coruña al convocar el concurso de proyectos de Ensanche de la ciudad, cumpliendo asi una disposición preceptiva del Estatuto Municipal, por un sistema 
- que facilita al máximo grado la concu() rrencia de ideas generales y la conjunción de parciales aciertos en términos de liberalidad técnica, que debieran ser incentivo y motivo de atracción de los profesionales, al tiempo mismo, que de gratitud al haberse ofrecido ocasión inmejorable de prueba para sus aptitudes, logro de sus aspiraciones y motivo de contribución al mejoramiento de España. Paralelamente, $y$ en sentido inverso, debe lamentarse el Jurado de no encontrar una digna respuesta de la técnica, a tan estimable invitación, y censurar que se abstengan cómodamente del esfuerzo que supone una competición, quienes entre sus aspiraciones tienen la de seguir una línea ascendente en su crédito profesional y por su pretendida especialidad debieran contribuir con sus conocimientos al fin principal perseguido en el concurso que hoy se juzga, cual es el de lograr una acertada solución a uno de los problemas urbanísticos que más pueden atraer e interesar por sus características e importancia a los técnicos españoles.

Estima el Jurado, en consecuencia del estudio de los cuatro proyectos antes citados, que las soluciones propuestas por los concursantes, no resuelven en conjunto los problemas de Ensanche y Extensión que tiene planteados el Ayuntamiento de La Coruña, y solo inician algunas soluciones parciales, $y$, con este criterio, $y$ el de estimar la colaboración auxiliar y el esfuerzo material que los concursantes han dedicado al proyectar los trazados con mayor o menor fortuna y acierto, se proponen:

Conceder el cuarto premio al proyecto suscrito por los Señores Arquitectos D. Joaquín García Alcañiz y D. Pedro Pigrau.

Conceder el quinto premio al proyecto suscrito por los Señores Arquitectos D. Ramiro Mariño Caruncho, D. Juan Martínez Barbeito y D. Pablo Iglesias Atocha.

De todo ello se levantó la presente acta, firmando de conformidad todos los asistentes y de la cual, como Secretario certifico. [...]

${ }^{15}$ GONZÁLEZ-CEBRIÁN, José. 1984. La Ciudad a través de su Plano. A Coruña, ( $1^{\mathrm{a} E d}$. Ayuntamiento de A Coruña, A Coruña), 174. 


\section{REFERENCIAS}

Ares Álvarez, Óscar. 2012. “La cuestión de la emulación y la importancia formal en el GATEPAC. El Concurso de Viviendas de Solocoeche." Proyecto, Progreso y Arquitectura 175: 174-183. https://doi.org/10.12795/ ppa.2012.i7.12

Argiz Vázquez, Emilio. 2004. “El Ferrol que pudo haber sido, el Proyecto de Reforma y ensanche de la población de Ferrol, de Santiago Rey Pedreira." Anuario Brigantino 27: 467-478.

García Estévez, Carolina. 2005. "El GATEPAC, GE. Una posible revisión historiográfica." DC PAPERS, revista de crítica y teoría de la arquitectura 13-14: 300-305.

García Fernández, Toni, and Yolanda Somoza Vales. 2008. Vivenda Colectiva, Vivenda Protexida, A Coruña: Edicións Espontáneas.

González-Cebrián, José. 1984. La Ciudad a través de su Plano. A Coruña. A Coruña: Ayuntamiento de A Coruña.

Gracia Alonso, Francisco. 2006. El sueño de una generación: el crucero universitario por el Mediterráneo de 1933. Barcelona: Publicacions i Edicions de la Universitat de Barcelona
Illescas de la Morena, Alberto. 2004. Sixte Illescas, Arquitecte. De la vanguardia al olvido. Barcelona: Col.legi d’Arquitectes de Catalunya.

López de Lucio, Ramón. 2013. Vivienda Colectiva, espacio público y ciudad. Evolución y crisis en el diseño de tejidos residenciales 1860-2010. Buenos Aires: Editorial Nobuko.

Rovira i Gimeno, Josep M. 2004. "Ordenar las vacaciones, diseñar el reposo. La Ciutat de Repós i de Vacances del GATCPAC en el litoral." Actas IV Congreso Fundación DOCOMOMO Ibérico: 35-46.

Sambricio Rivera-Echegaray, Carlos. 1976. "Ideologías y Reforma Urbana: Madrid 1920-1940." Arquitectura 199: 65-78.

Terán, Fernando de. 1978. Planeamiento Urbano en la España Contemporánea Historia de un Proceso Imposible. Barcelona: Gustavo Gili.

Urrutia Núñez, Ángel. 2002. "La vivienda obrera, GATEPAC." Arquitectura española contemporánea: documentos, escritos, testimonios inéditos: 218-219.

Vigo Trasancos, Alfredo. 2011. "El sueño urbano colectivista de un joven arquitecto coruñés." Quintana 10: 285-292. 
